\title{
Development of a Set of Benchmark Problems to Verify Numerical Methods for Solving Burnup Equations
}

Daniel Lago and Farzad Rahnema ${ }^{1}$

Nuclear \& Radiological Engineering and Medical Physics Programs

Georgia Institute of Technology

770 State Street NW

Atlanta, Georgia 30332-745, USA

dlago3@gatech.edu, farzad @gatech.edu

\begin{abstract}
A comprehensive set of transmutation chain benchmark problems for numerically validating methods for solving burnup equations was created. These benchmark problems were designed to challenge both traditional and modern numerical methods used to solve the complex set of ordinary differential equations used for tracking the change in nuclide concentrations over time due to nuclear phenomena. Given the development of most burnup solvers is done for the purpose of coupling with an established transport solution method, these problems provide a useful resource in testing and validating the burnup equation solver before coupling for use in a lattice or core depletion code. All the relevant parameters for each benchmark problem are described. Results are also provided in the form of reference solutions generated by the Mathematica tool, as well as additional numerical results from MATLAB.
\end{abstract}

\section{Keywords: burnup, benchmark, Bateman equations}

\section{Introduction}

Nuclear fuel undergoes constant and significant change during operation with criticality, radioactivity, and material performance all affected as a result. The formation of new isotopes following the absorption of a neutron in the fuel causes power shifts and flux profile changes; consequently, these phenomena need to be monitored to ensure safe and efficient reactor operation. Computationally, these events are simulated using lattice depletion codes - a catch-all term for the coupling of a neutron transport solver and a burnup equation solver.

Traditionally, burnup solvers are developed with the purpose of ultimately being coupled with an established neutronics solver. With very little in terms of standalone burnup solvers openly available, the lack of benchmarks to verify these solvers has become apparent. Before these burnup solvers can be used confidently to acquire the numerous parameters important to core life-cycle analysis, they must be thoroughly vetted to ensure sufficiently accurate solution methods.

\footnotetext{
${ }^{1}$ Corresponding author
} 
This study presents a set of benchmark problems developed for the verification of existing and new standalone burnup equation solvers. These benchmarks were specifically developed to challenge numerical solvers and help identify issues usually associated with burnup solver methods; e.g., matrix exponential methods and linear chain methods. The benchmarks are described in robust detail and results are presented for each problem as a resource for users to verify their own solvers and methods. The results include analytical solutions from the Mathematica package, as well as numerical results from MATLAB $[3,5]$.

\section{Development of Benchmark Problems for Numerical Verification of Burnup Solvers}

The benchmark problems in this study were developed to verify the numerical methods in a burnup solver. Starting with a simple two-isotope system and building up to a complex decay/transmutation chain, these benchmarks incorporate numerous physical phenomena associated with burnup problems and are designed to verify the accuracy of a solver trying to address these important events. Numerous decay chains were chosen, with and without reactions and fission yields, over a wide range of decay probabilities. The problems were chosen with the purpose of challenging the methods in burnup solvers to ensure accuracy of solutions for any given set of isotopes. Specifically, the isotopes and the time steps chosen generated highly illconditioned problems, meaning the condition number of the matrix corresponding to the problem is very large. As such, these matrices are close to singular, consequently making the computation of a solution prone to large numerical errors [4].

Additionally, these problems were generated for the explicit purpose of testing burnup solvers, not the neutronics solvers they are generally coupled with in lattice depletion codes; namely, the method for solving the matrix problem is tested, not the method of generating the matrix given a transport solution. The following subsection briefly describes the isotopes tracked in each of the transmutation chains. Section 3 details the specific parameters needed to regenerate the solution to each problem.

\subsection{Description of Benchmark Problems}

Benchmark problem \#1 is a simple decay scheme $-{ }^{238} \mathrm{U}$ alpha decays into ${ }^{234} \mathrm{Th}$, which then itself decays. While lacking complexity, this decay scheme tests any numerical method due to the wide range of half-lifes, leading to a highly ill-conditioned problem. 


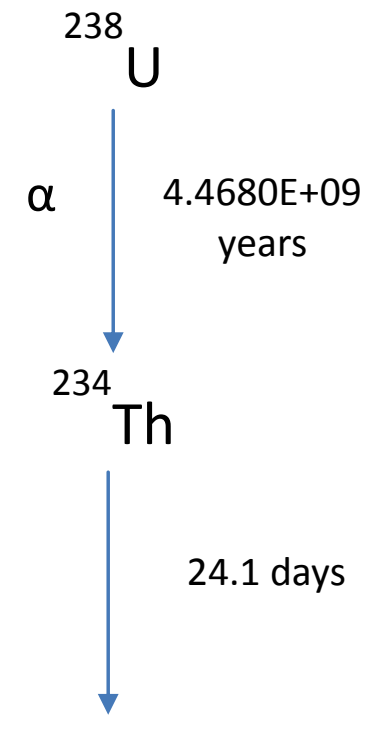

Figure 1. Description of Benchmark \#1.

Benchmark problem \#2 is another fairly simple decay scheme, but now introduces two types of decay - alpha decay and beta decay. This further tests the look-up schemes for the decay library in a burnup solver while still providing a wide range of eigenvalues to verify convergence to the correct solution.
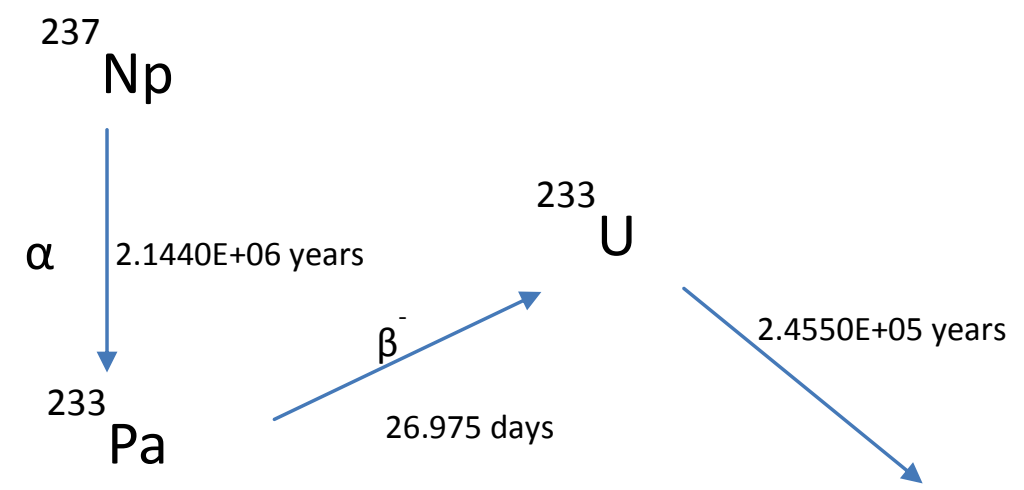

Figure 2. Description of Benchmark \#2.

Benchmark problem \#3 is similar to the first two benchmarks, but the introduction of ${ }^{207} \mathrm{~Pb}$ tests the ability of a matrix exponential method to identify and properly treat a stable isotope. In a matrix exponential method, a matrix of burnup coefficients called the transition matrix is generated. The transition matrix holds all the relevant parameters regarding the transmutation of each isotope - decay constant, reaction rate, etc. - and generally defines the condition of the problem. A stable isotope, such as ${ }^{207} \mathrm{~Pb}$, may introduce a zero element into the diagonal of the matrix, reducing the rank of the matrix to less than full and rendering the problem unsolvable. These elements need to be identified, reduced from the transition matrix, and solved for in a different manner. 


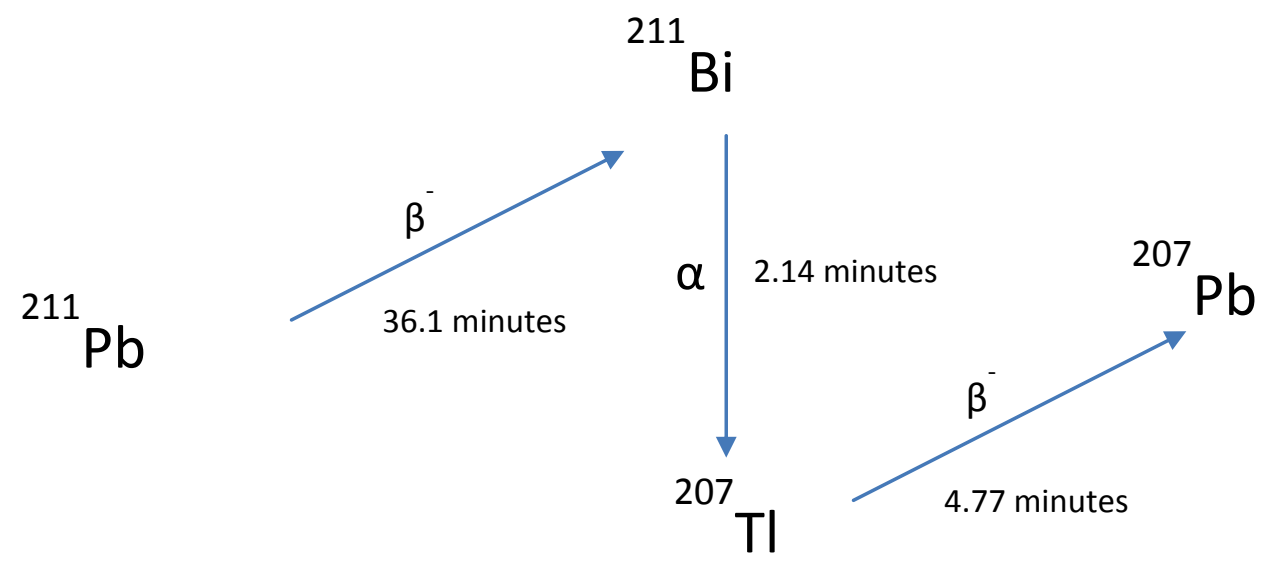

Figure 3. Description of Benchmark \#3.

Benchmark problem \#4 follows the production of ${ }^{237} \mathrm{~Np}$ via ${ }^{235} \mathrm{U}$. This problem includes the decay of each isotope, but now incorporates reaction rates - specifically the $(n, \gamma)$ reactions involved in producing ${ }^{237} \mathrm{~Np}$. This method tests the ability of a burnup solver to calculate and incorporate reaction rate branching ratios. Burnup solver algorithms need to be able to identify the relationship between each of the isotopes being tracked and properly implement the probability of one isotope decaying into another (branching ratios) via the information in decay libraries.

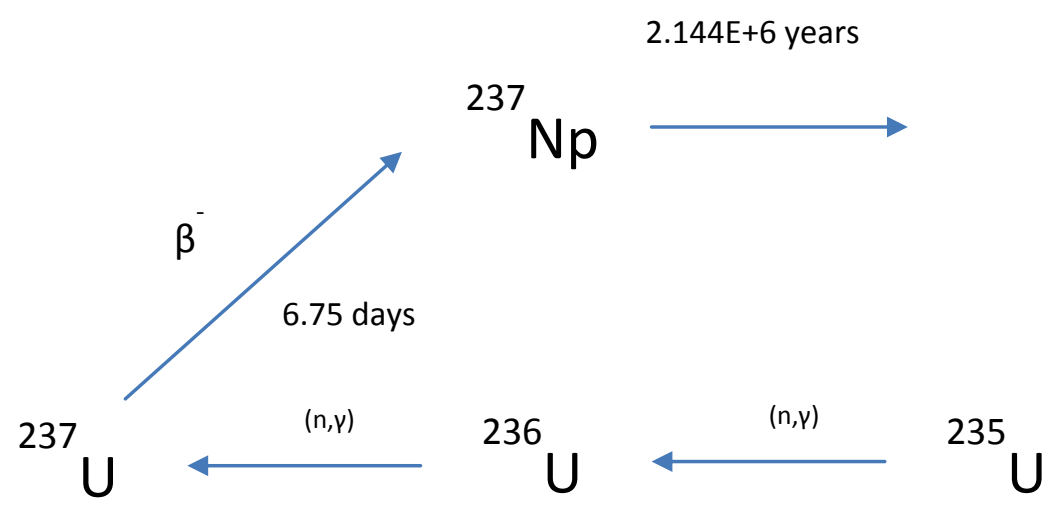

Figure 4. Description of Benchmark \#4.

Benchmark problem \#5 is a complex actinide chain following the decay and transmutation of ${ }^{238} \mathrm{U}$. This problem includes a wide range of decay probabilities, reaction rates, and a closed decay loop from ${ }^{244} \mathrm{Cm}$ to ${ }^{240} \mathrm{Pu}$. 


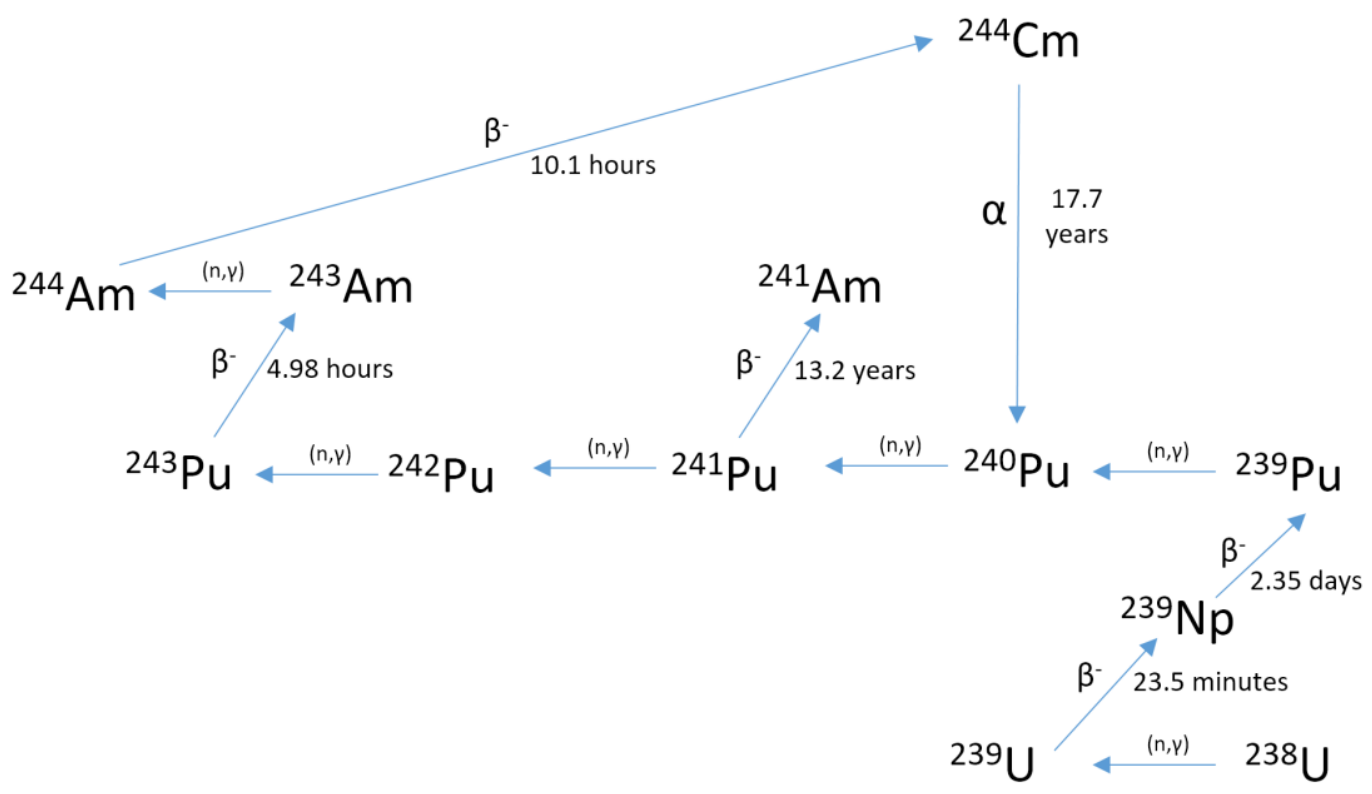

Figure 5. Description of Benchmark \#5.

Benchmark problem \# 6 is an analytical benchmark problem that follows the production of a fission product important to reactivity $-{ }^{135} \mathrm{Xe}$. This problem tests the methodology of calculating and incorporating fission product yields given an average incident neutron energy. Fissionable isotopes have fission yield probability data stored in the fission yield sublibrary of the ENDF/B libraries. For some isotopes, yields are available for multiple discrete energies of incident neutrons (thermal, epithermal and fast). Interpolating between these discrete energies is an important component of implementing fission yields. 


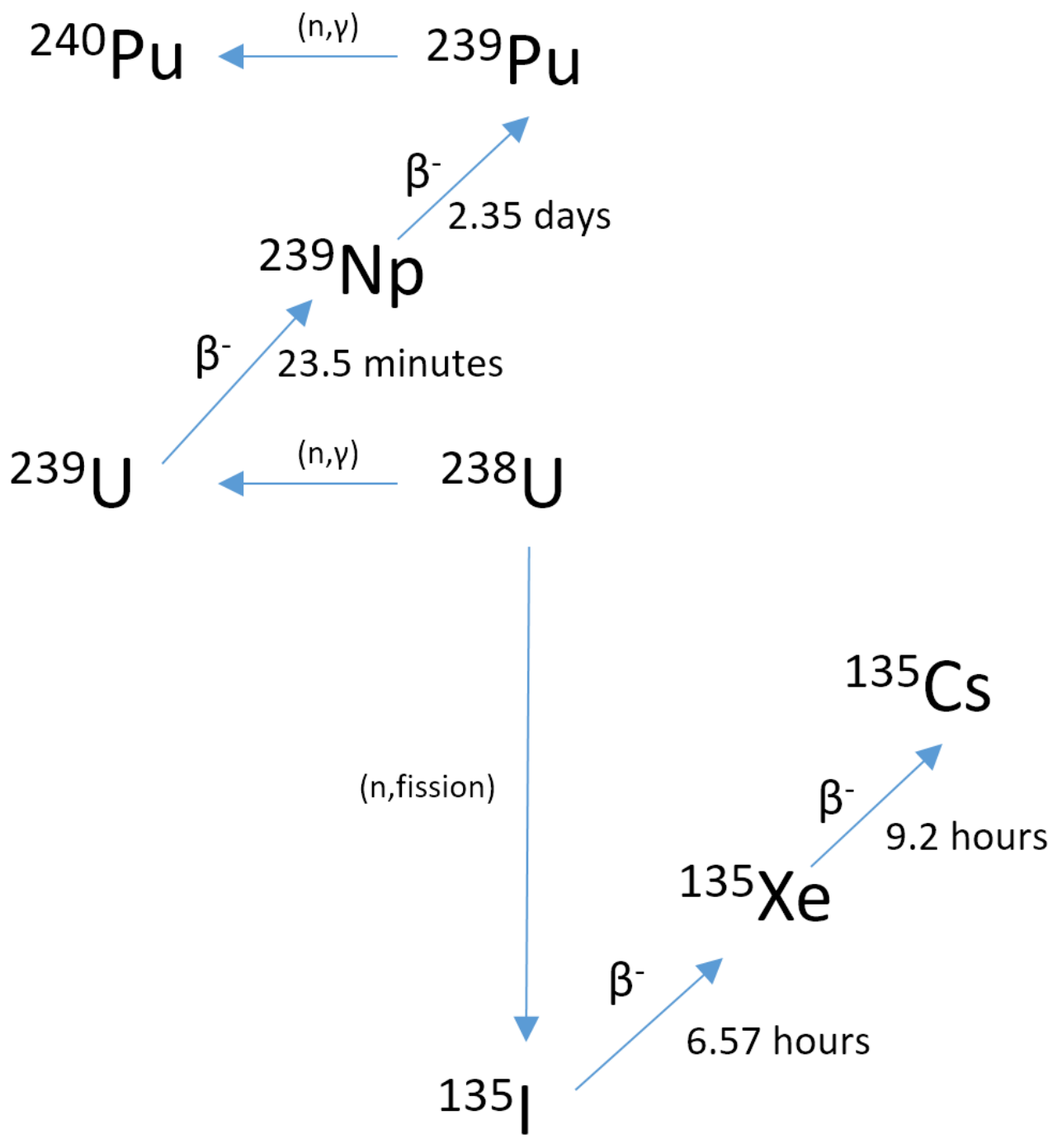

Figure 6. Description of Benchmark \#6. 


\subsection{Bateman Equations}

For each of the transmutation chains, an associated burnup equation is generated for each isotope in the chain. The collection of all the equations in each chain leads to a system of ordinary differential equations called the Bateman equations [1]. Generating these equations is straightforward, but for the sake of completeness, the general form for the time rate of change of nuclide concentration used in this study is presented below for the reader.

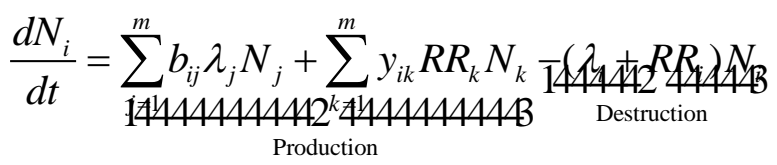

$$
\begin{aligned}
& R R_{i}=\int d X \phi(X) \sigma_{i}(X)
\end{aligned}
$$

$N_{i}=$ atom density of nuclide $i$

$\lambda_{i}=$ radioactive decay constant of nuclide $i$

$\sigma_{i}(X)=$ neutron absorption cross-section for nuclide $i$ over phase-space $X$

$\phi(X)=$ neutron angular flux over phase-space $X$

$b_{i j}=$ branching ratio of all other nuclides to nuclide $i$

$y_{i k}=$ branching ratio for neutron absorption by other nuclides that lead to nuclide $i$

\section{Parameters for Benchmark Problems}

For each benchmark problem, the chosen parameters - initial concentration, time step, reaction rates, etc. - reflect an extremely challenging set of conditions for numerical methods. Consequently, some of the parameters are indicative of a low-probability or non-physical event, but they are valid in a mathematical context. While not practical in a sense, these parameters provide a method of ensuring burnup solvers don't break down in any realistic scenario. Tables 1 through 6 detail the conditions for each problem. The initial concentration is given in atoms, the half-lifes are given in seconds, and the reaction rates are given in reactions per second. The nuclear data was acquired from the ENDF/B-VII.0 library [2]. Unless otherwise denoted, all decay branching ratios are 1.0 . 
Table 1. Parameters for Benchmark \#1.

Time step $($ seconds $)=5.00 \mathrm{E}+17$

Initial Concentration (atoms) Half-life (sec)

\begin{tabular}{|c|c|c|}
\hline U-238 & $1.0000 \mathrm{E}+10$ & $1.4099935680 \mathrm{E}+17$ \\
\hline Th-234* & $0.0000 \mathrm{E}+00$ & $2.082240 \mathrm{E}+06$ \\
\hline
\end{tabular}

Table 2. Parameters for Benchmark \#2.

Time step $($ seconds $)=1.00 \mathrm{E}+12$

Initial Concentration (atoms) Half-life (sec)

\begin{tabular}{|c|c|c|}
\hline Np-237 & $1.00 \mathrm{E}+12$ & $6.7659494310 \mathrm{E}+13$ \\
\hline Pa-233 & $0.00 \mathrm{E}+00$ & $2.330640 \mathrm{E}+06$ \\
\hline U-233 & $0.00 \mathrm{E}+00$ & $5.023969920 \mathrm{E}+12$ \\
\hline
\end{tabular}

Table 3. Parameters for Benchmark \#3.

Time step $($ seconds $)=1.00 \mathrm{E}+04$

Initial Concentration (atoms) Half-life (sec)

\begin{tabular}{|c|c|c|}
\hline Pb-211 & $1.00 \mathrm{E}+10$ & $2.1660 \mathrm{E}+03$ \\
\hline Bi-211 & $1.00 \mathrm{E}+04$ & $1.2840 \mathrm{E}+02$ \\
\hline Tl-207 & $1.00 \mathrm{E}+01$ & $2.8620 \mathrm{E}+02$ \\
\hline Pb-207 & $0.00 \mathrm{E}+00$ & Stable \\
\hline
\end{tabular}

Table 4. Parameters for Benchmark \#4.

Time step $($ seconds $)=8.64 \mathrm{E}+04$

\begin{tabular}{|c|c|c|c|}
\multicolumn{1}{c}{ Initial Concentration (atoms) } & \multicolumn{1}{c|}{ Half-life (sec) } & $(\mathbf{n}, \boldsymbol{\gamma})$ Rxn Rate (sec ${ }^{-1}$ ) \\
\hline U-235 & $1.00 \mathrm{E}+12$ & $2.2210238880 \mathrm{E}+16$ & $1.0 \mathrm{E}-04$ \\
\hline $\mathbf{U - 2 3 6}$ & $1.00 \mathrm{E}+02$ & $7.390789920 \mathrm{E}+14$ & $1.0 \mathrm{E}-04$ \\
\hline $\mathbf{U - 2 3 7}$ & $1.00 \mathrm{E}+02$ & $5.8320 \mathrm{E}+05$ & - \\
\hline Np-237 & $1.00 \mathrm{E}+02$ & $6.7659494310 \mathrm{E}+13$ & - \\
\hline
\end{tabular}


Table 5. Parameters for Benchmark \#5.

Time step (seconds) $=8.64 \mathrm{E}+05$

\begin{tabular}{|c|c|c|c|}
\multicolumn{1}{c}{ Initial Concentration (atoms) } & \multicolumn{1}{c|}{ Half-life (sec) } & $(\mathbf{n}, \boldsymbol{\gamma})$ Rxn Rate $\left(\mathbf{s e c}^{-1}\right)$ \\
\hline U-238 & $1.00 \mathrm{E}+10$ & $1.4099935680 \mathrm{E}+17$ & $1.0 \mathrm{E}-04$ \\
\hline U-239 & $1.00 \mathrm{E}+03$ & $1.4070 \mathrm{E}+03$ & - \\
\hline Np-239 & $0.00 \mathrm{E}+00$ & $2.0355840 \mathrm{E}+05$ & - \\
\hline Pu-239 & $0.00 \mathrm{E}+00$ & $7.60853735110 \mathrm{E}+11$ & $1.0 \mathrm{E}-04$ \\
\hline Pu-240 & $0.00 \mathrm{E}+00$ & $2.0704941360 \mathrm{E}+11$ & $1.0 \mathrm{E}-04$ \\
\hline Pu-241 & $0.00 \mathrm{E}+00$ & $4.509581040 \mathrm{E}+08$ & $1.0 \mathrm{E}-04$ \\
\hline Pu-242 & $0.00 \mathrm{E}+00$ & $1.178676360 \mathrm{E}+13$ & $1.0 \mathrm{E}-04$ \\
\hline Pu-243 & $0.00 \mathrm{E}+00$ & $1.784160 \mathrm{E}+04$ & - \\
\hline Am241 & $0.00 \mathrm{E}+00$ & $1.3651817760 \mathrm{E}+10$ & - \\
\hline Am-243 & $0.00 \mathrm{E}+00$ & $2.325795120 \mathrm{E}+11$ & $1.0 \mathrm{E}-04$ \\
\hline Am-244 & $0.00 \mathrm{E}+00$ & $3.6360 \mathrm{E}+04$ & - \\
\hline Cm-244 & $0.00 \mathrm{E}+00$ & $5.715081360 \mathrm{E}+08$ & - \\
\hline
\end{tabular}

Table 6. Parameters for Benchmark \#6.

Time step $($ seconds $)=1 \mathrm{E}+05$

\section{Initial \\ Concentration Half-life (sec) (n, $\quad$ Rxn Rate $\left(\mathrm{sec}^{-1}\right) \quad$ Rate $_{\left(\mathrm{sec}^{-1}\right)}$ (atoms)}

\begin{tabular}{|c|c|c|c|c|}
\hline U-238 & $1.00 \mathrm{E}+12$ & $1.4099935680 \mathrm{E}+17$ & $1.0 \mathrm{E}-04$ & $1.0 \mathrm{E}-05^{*}$ \\
\hline U-239 & $0.00 \mathrm{E}+00$ & $1.4070 \mathrm{E}+03$ & - & - \\
\hline Np-239 & $0.00 \mathrm{E}+00$ & $2.0355840 \mathrm{E}+05$ & - & - \\
\hline Pu-239 & $0.00 \mathrm{E}+00$ & $7.60853735110 \mathrm{E}+11$ & $1.0 \mathrm{E}-05$ & - \\
\hline Pu-240 & $0.00 \mathrm{E}+00$ & $2.0704941360 \mathrm{E}+11$ & - & - \\
\hline I-135 & $0.00 \mathrm{E}+00$ & 23652 & - & - \\
\hline Xe-135 & $0.00 \mathrm{E}+00$ & $3.29040 \mathrm{E}+04$ & - & - \\
\hline Cs-135 & $0.00 \mathrm{E}+00$ & $7.2582480 \mathrm{E}+13$ & - & - \\
\hline
\end{tabular}

*The fission branching ratio of U-238 to Xe-135 is $1.11541594 \mathrm{E}-04$ (at $1.0 \mathrm{eV}$ )

\section{Results}

The results (final concentration in number of atoms) for each benchmark problem are presented in Tables 7 through 12. For each problem, the generated results from Mathematica using the 'DSolve' function are shown as the reference solutions. Additionally, the results generated from the matrix exponential function in MATLAB ('expm') are also presented for the reader. Note that while the results for Th-234 in benchmark \# 1 are non-physical due to the 
context of the problem, the mathematical rigor is correct and valid in terms of verifying numerical results.

Table 7. Final concentrations (atoms) for Benchmark \#1.

\begin{tabular}{|c|c|c|}
\hline \multicolumn{1}{c}{ Mathematica } & MATLAB \\
\hline U-238 & 856027125 & 856076082 \\
\hline Th-234 & 0.012641889 & 0.012642298 \\
\hline
\end{tabular}

Table 8. Final concentrations (atoms) for Benchmark \#2.

\begin{tabular}{|c|c|c|}
\multicolumn{1}{c}{ Mathematica } & MATLAB \\
\hline Np-237 & 989807657321 & 989807657316 \\
\hline Pa-233 & 34096 & 34096 \\
\hline U-233 & 9519333638 & 9519333605 \\
\hline
\end{tabular}

Table 9. Final concentrations (atoms) for Benchmark \#3.

\begin{tabular}{|c|c|c|}
\multicolumn{1}{c}{ Mathematica } & MATLAB \\
\hline Pb-211 & 407570893 & 407570892 \\
\hline Bi-211 & 25683207 & 25683207 \\
\hline Tl-207 & 65963040 & 65780982 \\
\hline Pb-207 & 9500792870 & 9500782861 \\
\hline
\end{tabular}

Table 10. Final concentrations (atoms) for Benchmark \#4.

\begin{tabular}{|c|c|c|c|}
\multicolumn{1}{c}{} & Mathematica & MATLAB & APIDA \\
\hline U-235 & 176886799 & 176886902 & 176886902 \\
\hline U-236 & 1528302359 & 1528302835 & 1528302835 \\
\hline U-237 & 922519446486 & 922519445898 & 922519445898 \\
\hline Np-237 & 75775364619 & 75775364627 & 75775364627 \\
\hline
\end{tabular}


Table 11. Final concentrations (atoms) for Benchmark \#5.

\begin{tabular}{|c|c|c|}
\multicolumn{1}{c}{} & Mathematica & MATLAB \\
\hline U-238 & 1768869 & 1768869 \\
\hline U-239 & 450504 & 450504 \\
\hline Np-239 & 7765299776 & 7765299776 \\
\hline Pu-239 & 273085486 & 273085486 \\
\hline Pu-240 & 279959954 & 279959954 \\
\hline Pu-241 & 282115515 & 282115515 \\
\hline Pu-242 & 21477 & 21477 \\
\hline Pu-243 & 275858289 & 275858289 \\
\hline Am-241 & 529647302 & 529647302 \\
\hline Am-243 & 172381411 & 172381411 \\
\hline Am-244 & 312220315 & 312220315 \\
\hline Cm-244 & 107192026 & 107192026 \\
\hline
\end{tabular}

Table 12. Final concentrations (atoms) for Benchmark \#6.

\begin{tabular}{|c|c|c|}
\multicolumn{1}{c}{} & Mathematica & MATLAB \\
\hline U-238 & 16701722 & 16701701 \\
\hline U-239 & 4364844 & 4364838 \\
\hline Np-239 & 672015319314 & 672015319330 \\
\hline Pu-239 & 153111500325 & 153111500345 \\
\hline Pu-240 & 83944524243 & 83944524231 \\
\hline I-135 & 85435214 & 85435214 \\
\hline Xe-135 & 299196873 & 299196873 \\
\hline Cs-135 & 796828259 & 796828259 \\
\hline
\end{tabular}

\section{Conclusions}

In this paper, a set of benchmark problems was created to validate numerical methods for solving the burnup (Bateman) equations. Six problems were generated, all built to challenge numerical solvers for accuracy. These problem descriptions in this paper are sufficiently selfcontained such that the reader can reproduce these reference solutions in any mathematical solver suite, such as Mathematica. For the convenience of the reader, this paper provides those reference solutions as well as numerical results from MATLAB. Future work includes the 
generation of more problems to test additional features present in burnup solvers. Specifically, tests should be generated in conjunction with explicit transport solutions to ensure the proper generation of transition matrices.

\section{Acknowledgements}

The first author would like to acknowledge this research is being performed using funding received from the U.S. Department of Energy Office of Nuclear Energy's Nuclear Energy University Programs. Any opinions, findings, conclusions, or recommendations expressed in this publication are those of the authors and do not necessarily reflect the views of the U.S. Department of Energy Office of Nuclear Energy.

\section{References}

1. Bateman, H. Proc. Cambridge Phil. Soc., no. 15, p. 423, 1910.

2. Chadwick, M. B. and others, "ENDF/B-VII.0: Next Generation Evaluated Nuclear Data Library for Nuclear Science and Technology," Nuclear Data Sheets, vol.107, pp. 29313060, 2006.

3. MATLAB version 7.14.0. Natick, Massachusetts: The MathWorks Inc., 2012.

4. Moler, C., Van Loan, C., "Nineteen Dubious Ways to Compute the Exponential of a Matrix, Twenty-Five Years Later," SIAM Rev., 45 (2003).

5. Wolfram Research, Inc., Mathematica, Version 10.4, Champaign, IL (2016). 